\title{
MCTAGGART, SCHLESINGER, AND THE TWO-DIMENSIONAL TIME HYPOTHESIS
}

\author{
By L. NATHAN OAKLANDER
}

In "The Unreality of Time" and The Nature of Existence, McTaggart offers a positive conception of time and then provides arguments that purport to establish that such a concept is contradictory and therefore cannot be applied to reality $([7],[8])$. Although there are few, if any, philosophers who agree with McTaggart's conclusion that time is unreal, there are many who accept the general position that time involves passage or tense (cf. [2], [4], [6], [10], [11]). George Schlesinger is one philosopher sympathetic with McTaggart's positive views on time and in his recent book, Aspects of Time, he attempts to defend McTaggart's account of the passage of time ([14], pp. 30-33) by resuscitating a gambit suggested some time ago by C. D. Broad ([1], [3]; cf. [9]), namely, the notion that time has two dimensions. The purpose of this paper is to argue that Schlesinger does not vindicate MacTaggart's positive conception of time, since the two-dimensional time hypothesis that it allegedly requires, is as beset with difficulties as the conception of temporal becoming it is supposed to render intelligible.

It will be useful for us to begin by stating the main elements of Schlesinger's interpretation of McTaggart's positive account of time since it is the view that he (hesitantly) intends to defend. According to McTaggart, the NOW is something that moves relative to the series of points that constitute time. Schlesinger continues:

2 I am grateful to Professor Hare for many helpful comments and suggestions. 
Temporal points from the future, together with the events that occur at those points, keep approaching the NOW and after momentarily coinciding with it they recede further and further into the past. The NOW is, of course, not conceived as some sort of object but rather as the point in time at which any individual who is temporally extended is alive, real or Exists with a capital $\mathrm{E}$. ... A typical event, on this view, to begin with is in the distant future; then it becomes situated in the less distant future; it keeps approaching us until it becomes an event occurring in the present. As soon as this happens the event loses its presentness and acquires the property of being in the near past ([14], p. 23).

(For other recent interpretations of McTaggart on time see Christensen and Rankin [5], [12]). Schlesinger also mentions with approval Gale's characterization of the NOW as a moving spotlight which successively illuminates different moments along the series of time ([14], p. 132). Several major objections have been made to this way of conceiving of the moving NOW, but Schlesinger believes that they can be answered.

One such objection derives from J. J. C. Smart. Smart argues that if we think of time as a river, or some kind of particular thing which moves, then it must make sense to ask, How fast is it moving? Yet the question, How fast did time flow yesterday? seems to be a senseless question. Smart says:

We do not know how we ought to set about answering it. What sort of measurements ought we to make? We do not even know the sort of units in which our answer should be expressed ([15], p. 485).

Since we cannot measure the speed of the NOW, there is reason to suppose that it does not exist.

A second and stronger argument derives from Broad ([2], pp. 277-280). Suppose that the NOW moves along a series of events. When the NOW "hits" a given event $e_{1}$, that event acquires the property of presentness and then loses it without delay. Although the acquisition and subsequent loss of presentness is itself an event; that event, i.e., $e_{1}$ 's acquirement of presentness, cannot be a member of the very set which constitutes the first series of events. Thus, Broad concludes that if we accept the moving NOW, then we must postulate a second time-dimension in which events of the first time-dimension acquire and lose presentness. As Broad puts it,

If there is any sense in talking of presentness moving along a series of events, related by the relation of earlier-and-later, we must postulate a second timedimension in addition to that in which the series is spread out. An event which has zero duration, and therefore no history, in the first time-dimension, will yet have an indefinitely long duration and a history in the second timedimension ([2], p. 278).

If one finds the notion of a second time-dimension unintelligible then one will reject the conception of the moving NOW that leads to it.

Schlesinger claims that this objection to the moving NOW is virtually identical with McTaggart's main argument for the unreality of time. The difficulty arose because in a world of absolute becoming one and the same instantaneous event has the incompatible characteristics of pastness, presentness and futurity. This, however, is impossible because incompatible properties can only be possessed by the same entity if it has them 
at different times. Yet, events do not have any temporal scope during which they could accommodate incompatible properties. The "way out" of this difficulty, and of the preceding one, is to postulate a second higher order series of meta-moments in which literally instantaneous events have an indefinite duration. Schlesinger calls this idea "fanciful" but he does seriously consider it as a way of defending McTaggart's conception of the transient aspect of time and resolving McTaggart's paradox.

According to Schlesinger, McTaggart's conception of time can be made intelligible, and its difficulties overcome if we agree to postulate a higher order series of moments. He answers Smart's objection as follows:

The movement of the NOW in the standard series of time may be explicated by explaining that the NOW is at $t_{1}$ in the ordiary series when it is at $T_{1}$ in the super-series, and at $t_{2}$ in the ordinary series when it is at $T_{2}$ in the superseries. We may even assign a value to the speed of the NOW: it moves from $t_{1}$ to $t_{2}$ at the average speed of

$$
\frac{t_{1}-t_{2}}{T_{1}-T_{2}}([14], \text { p. 32). }
$$

Concerning Broad's objection, Schlesinger says that "the event of the NOW reaching $t_{1}$ may if we like be looked on as taking place in super-time" (p. 32). He is quite naturally reluctant to postulate a whole new series of temporal points, but is comforted by his belief that we need not continually postulate a new time series to help us make sense of temporal becoming in the preceding one.

For just as the second series could be instrumental in helping to make sense of temporal becoming in the first series, in the same manner the first series could serve as the extra series through the use of which temporal becoming in the second series could make sense. For example, it might be said that the NOW in the second series is at $\mathrm{T}^{\prime}{ }_{1}$ when it is at $\mathrm{t}^{\prime}{ }_{1}$ in the first series, and at $\mathrm{T}^{\prime}{ }_{2}$ when it is at $\mathrm{t}^{\prime}{ }_{2}$, leading to the claim that the average speed of the NOW in the second series from

$$
\mathrm{T}_{1}{ }^{\prime} \text { to } \mathrm{T}_{2}{ }^{\prime} \text { is } \frac{\mathrm{T}_{1}{ }^{\prime}-\mathrm{T}_{2}{ }^{\prime}}{\mathrm{t}_{1}{ }^{\prime}-\mathrm{t}_{2}{ }^{\prime}} \text { (p. 32). }
$$

Finally, Schlesinger uses a second-order time series to resolve McTaggart's difficulty concerning A-characteristics. On the two-dimensional time hypothesis,

All the moments of our regular time series co-exist together at each moment in super-time, and the position of the NOW in regular time varies from moment to moment in super-time. $M_{1}$ and every other moment in regular time can assume different properties at different moments in super-time.... Thus, the problem of the extensionlessness of the moments in the continuum of which they form a part is resolved with the introduction of a higher order time continuum in which they have unlimited duration (pp. 140-141).

Can this account of the movement of time be accepted as a way of avoiding paradox and resolving traditional philosophical perplexities concerning time? Let us see.

Schlesinger offers a challenge to those who would attempt to refute McTaggart's 
claim that the NOW is a particular which shifts its position relative to the series of events in the direction of the future. He says,

First, it would have to be shown that McTaggart cannot make sense of the changes going on in ordinary life unless he postulates a meta-time of equal richness. That is, a meta-time that admitted B-relations only would not be capable of performing its required function. Secondly, it would have to be shown that in order to explicate the movement of the NOW in meta-time, we could not employ standard time in the same manner we employed meta-time to explicate the movement of the NOW in standard time and therefore we would be forced to introduce a third temporal series. Lastly, it would have to be shown why the regress thus resulting would have to be regarded as vicious (pp. 32-33).

It seems to me that three things that would have to be shown in order to refute McTaggart's conception of the transient aspect of time can indeed be shown, and in what follows I shall attempt to explain why.

Turning to the first issue: Is it possible for the terms of the original series to undergo genuine change, in McTaggart's sense, if the terms of meta-time form a series that have B-relations (earlier and later) but do not have A-determinations (pastness, presentness, and futurity)? The answer to this question is emphatically no. McTaggart is very explicit in his belief that the B-series has no independent and separate reality, but is entirely dependent and inseparable from the application of the A-series to the non-temporal $\mathrm{C}$-series. According to McTaggart, you cannot have B-relations between events unless those events have A-determinations and change with respect to them. As further evidence consider the following passage:

If there is any change, it must be looked for in the A-series ... If there is no real A-series, there is no real change. The B-series, therefore, is not by itself sufficient to constitute time, since time involves change. The B-series, however, cannot exist except as temporal since earlier and later, which are the relations which connect its terms, are clearly time-relations. So it follows that there can be no $B$-series when there is no $A$-series, since mithout an $A$-series there is no time ([8], p. 13).

If it was possible for there to be genuinely temporal relations between terms without those terms having A-determinations, then it would be possible for there to be succession (a genuinely temporal series) without becoming. Clearly, McTaggart would not accept that conclusion, and since Schlesinger claims to be in complete agreement with McTaggart's positive conception of time and change, he could not accept it either.

Thus a second series that admitted B-relations only would not be capable of performing its required function because it would not be a temporal series at all. Consequently, an event could not possibly endure indefinitely in the second series. Indeed, it could not endure at all since endurance requires time and in a B-series without an A-series there is no time. To put the point still differently, since a B-series without an A-series is not a temporal series, the second series without becoming does not enable the first series to avoid the incompatible properties problem. Thus it would appear that McTaggart cannot make sense of the changes going on in ordinary time unless he postulates a meta-time of equal richness. 
Turning to the second issue, the pertinent question is as follows: Can the movement of the NOW in meta-time be explained by reference to standard time in the same manner in which reference to meta-time is employed to explicate the movement of the NOW in standard time? Again, it seems to me that the answer is no, and to see why let us first note that the movement of the NOW in standard time is never made clear. According to Schlesinger, the moving NOW in the first series is explicated by saying that "the NOW is at $t_{1}$ in the ordinary series when it is at $T_{1}$ in the super-series, and at $t_{2}$ in the ordinary series when it is at $T_{2}$ in the super-series" (p. 32). There are, however, serious problems with this first step. Since Schlesinger maintains that the events that are at the point in time at which the NOW is situated are those that are real and alive, or exist with a capital $\mathrm{E}$, and since he also maintains that when the NOW is situated at $\mathrm{t}_{1}$ it is also situated at $T_{1}$, it follows that when the NOW is at $t_{1}$ not only do the events at $t_{1}$ Exist with a capital $\mathrm{E}$, but also the events that are at $\mathrm{T}_{1}$ Exist with a capital $\mathrm{E}$. Furthermore, since Schlesinger claims that "all the moments of our regular time series co-exist together at each moment in super-time", it follows that when the events at $\mathrm{t}_{1}$ are NOW, the events at $T_{1}$, that is, the events at $t_{1}, t_{2}, \ldots t_{n}$, are also NOW, and that is absurd. For if the events in the original series are all NOW at $T_{1}$ in the second series, then they exist simultaneously and not successively. In other words, this account of the moving NOW does not make sense of time and change in the first series. It eliminates it!

My objection to Schlesinger's account of the moving NOW in the first series can be approached from a different direction. When the NOW is at $t_{1}$ in the original series, neither the past events at $t_{1-n}$ nor the future ones at $t_{1+n}$ exist. For events of the past do not exist now but only in the past, and "the future has no reality by means of which to reach out toward us and make an impact on the present" (p. 60). On the other hand, when the NOW is at $t_{1}$ it is also at $T_{1}$, and when it is at $T_{1}$ all the moments and events in the first time series co-exist. If, however, they all co-exist, then they cannot be distinguished as past, present, and future, but they must all be present, and that is unacceptable because: (1) it entails that past and future moments both do not exist (at the first level) and do exist (at the second level); (2) Since past and future events are NOW, they exist simultaneously with those that are present, and hence there is no ground for the original series being temporal; (3) Since the moments that are NOW at

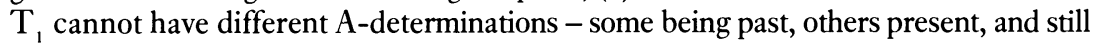
others future - and then change those determinations when the NOW is at $\mathrm{T}_{2}$, the appeal to a second time-dimension does not, despite initial appearances, help resolve McTaggart's difficulties.

Having said so much, we can deal briefly with the question of whether or not we can understand the moving NOW in the second series by employing standard time. Recall, meta-time consists of a temporal series of moments the contents of which are whole series of moments of original time. If the second series is to be a genuinely temporal series, then its terms must change with respect to their A-determinations and the question arises as to how this is possible. Could it be that each term $T_{i}$ which has a momentary existence in the second series has an indefinite history in the first series and thus changes properties at different $t_{i}$ 's in the first series? This would make no sense whatsoever, for if it were true, then each $t_{i}$ would contain the contents of every term in the second series. Consequently, all events in the first series would exist at each $t_{i}$, and that contradicts the original presupposition that the terms of standard time do not all exist at the same $t_{i}$. Furthermore, if all the terms in the second series co-exist in each moment of the first, and if we say, as Schlesinger does, that the NOW is at $T_{1}$ when it is 
at $t_{1}$ then it would follow that if one of the terms of the second series is NOW, then all of the terms of the second series are NOW. But then, the second series, like the first, no longer deserves to be called temporal. It would appear then, that the movement of the NOW in the second time dimension cannot be understood in terms of the movement of the NOW in the original time dimension, but would require the postulation of a third time series, a fourth and so on.

We are thus lead to the third and final question: Does the resulting regress have to be regarded as vicious? Here I think that the answer is yes, for at no stage along the regress of time series can we stop and say that the questions and problems for which they were introduced have been answered or solved. In other words, the infinite regress of time dimensions is vicious because the notion that we are attempting to understand by an appeal to a higher order time series arises in exactly the same form in that higher order series, and consequently, regardless of how many time dimensions we introduce we never manage to answer the problem for which they were introduced. More specifically, the problem of time and change centres around the following question: How are we to understand the common-sense belief that an apple is green at one time and red at a later time, or equivalently, an apple is green before it is red? What must time be in order for it to be possible for a single entity to have a property and then lose it? Presumably, the account of time developed by Schlesinger and McTaggart is intended to answer that question. Their answer involves the notion of the NOW moving along a series of events such that it is at the point $\left(t_{1}\right)$ at which the green temporal slice of the apple exists at one time $\left(\mathrm{T}_{1}\right)$ and it is at the point $\left(\mathrm{t}_{2}\right)$ at which the red temporal slice of the apple exists at another time $\left(\mathrm{T}_{2}\right)$. Alternatively, they could say that the NOW is simultaneous with the green slice before it is simultaneous with the red slice of the apple. As Schlesinger says, it is useful to think of the NOW as a moving spotlight which illuminates successively different moments along the series of time. Unfortunately, this account does not help us to understand the nature of time and change because it presupposes it. For Schlesinger is treating the NOW as a substance that has a property at one time then loses it at another time (it is first simultaneous with the green section and then it is later than the green section), and we want to know how this is possible. Thus, the moving NOW involves precisely the same notion that we hoped to make intelligible, thereby falling into a vicious circle.

The circularity of Schlesinger's solution to the problem of time can be seen in still a different way. For Schlesinger, the account of an ordinary substance changing from green to red is explicated in terms of qualitatively different temporal cross-sections of events (e.g., the green section and the red section) which comprises that substance changing from future to present to past. Such change he claims is unintelligible unless temporal slices of substances have an indefinite duration in a second time-dimension and exemplify different properties at different points in that second series. But then we are faced with the original question at the second level: How can a single thing (say, the red section) of an indefinitely long duration have a property (say futurity) at one time and then not have it at another time? By parity of reasoning, Schlesinger must admit that change in the red section of the second series is to be understood in terms of temporal slices (e.g., the red sections being present, the red sections being past, etc.) that undergo becoming. Thus, to "solve" the problem of change in the second series we must appeal to a third time-dimension in which an instantaneous event in the second series, say, the red section's being present has an unlimited duration and exemplifies different A-properties at different times. It should by now be evident, however, that, 
and why, such a move is futile. Thus, the infinite regress of time dimensions is a vicious one because at each level the concept which is was introduced to comprehend remains incomprehensible.

The three things which Schlesinger claims would have to be done in order to refute Mc Taggart's account of the moving NOW in terms of a meta-time can be done, as I have shown. Thus, the hypothesis that there exists a second time-dimension neither aids in our understanding of the passage of time nor resolves McTaggart's difficulties concerning that notion. I conclude, therefore, that Schlesinger has not provided an adequate defense of McTaggart's positive conception of time. ${ }^{1}$

The University of Michigan-Flint

\section{REFERENCES}

[1] C. D. Broad, "The philosophical implications of foreknowledge", Aristotelian Soc. Supp. 16 (1937), 177-209.

[2] C. D. Broad, Examination of McTaggart's Philosophy, II, I (Cambridge, 1938).

[3] C. D. Broad, "A reply to my critics," in [13], 711-830.

[4] R. D. Chisholm, The First Person (Minnesota, 1981).

[5] F. Christensen, "McTaggart's paradox and the nature of time", The Philosophical Quarterly 24 (1974), 289-299.

[6] R. Gale, The Language of Time (New York, 1968).

[7] J. M. E. McTaggart, “The unreality of time”, Mind 18 (1908), 457-484.

[8] J. M. E. McTaggart, The Nature of Existence, II, ed. C. D. Broad (Cambridge, 1927).

[9] H. H. Price, "The philosophical implications of precognition", Aristotelian Soc. Supp. 16 (1937), pp. 211-228.

[10] A. N. Prior, Past, Present and Future, (Oxford, 1967).

11] A. N. Prior, Time and Tense, (Oxford, 1968).

[12] K. Rankin, "McTaggart's paradox: two parodies", Philosophy 56 (1981), 333348.

[13] P. A. Schilpp, ed., The Philosophy of C. D. Broad, (New York, 1959).

[14] G. Schlesinger, Aspects of Time, (Indianapolis, 1980).

[15] J. J. C. Smart, "The river of time”, Mind 58 (1949), 481-494.

1 This paper was funded by a Faculty Development Grant, The University of Michigan-Flint. 